\title{
Autonomic Denervation Hypersensitivity in the Primary Glaucomas
}

\author{
CHARLES V. CLARK \\ Edinburgh
}

\begin{abstract}
Summary
Autonomic denervation hypersensitivity of the iris was assessed in 44 patients with primary closed-angle glaucoma, 20 patients with primary open-angle glaucoma, and 40 age- and sex-matched control subjects. Significantly increased pupillary constriction in response to $2.5 \%$ methacholine chloride, indicating parasympathetic denervation hypersensitivity, was present in both closed-angle $(p<0.02)$ and openangle glaucoma patients $(p<0.001)$, compared with the control group. Significantly increased pupillary dilatation in response to 0.5 per cent phenylephrine hydrochloride, indicating sympathetic denervation hypersensitivity of the iris, was also present in both closed-angle glaucoma $(p<0.01)$ and open-angle glaucoma patients $(p<0.05)$. The association between autonomic neuropathy and the primary glaucomas is discussed, with particular reference to the potential effects of autonomic dysfunction on aqueous dynamics.
\end{abstract}

Elevation of intraocular pressure in the primary glaucomas occurs as a consequence of impaired outflow of aqueous humour from the anterior chamber of the eye. The mechanism of obstruction to outflow forms the basis of categorisation in glaucoma: in closed-angle glaucoma the exit of aqueous from the anterior chamber is physically prevented by apposition of peripheral iris to cornea; in open-angle glaucoma the site of obstruction lies within the angle, probably the trabecular meshwork, and the entrance to the angle is not a primary contributing factor to the glaucomatous process. The pathological effects of raised intraocular pressure on the retinal nerve fibre layer are well established, however the aetiological factors precipitating the primary glaucomas have not been fully defined. Autonomic nerve function is a major determinant of intraocular pressure; glaucoma therapeutics are based upon manip- ulation of the autonomic nervous system in the anterior segment of the eye. The efficacy of ocular autonomic nerve function can be inferred from the assessment of denervation hypersensitivity. In the presence of autonomic neuropathy, receptor density is significantly increased at the postsynaptic receptor site, ${ }^{1}$ and may be detected by the measurement of exaggerated end-organ responses to specific autonomic agonists. The pupil provides an excellent model for the assessment of autonomic integrity in the eye; pupillary diameter represents a balance between parasympathetic and sympathetic activity in the anterior segment, and assessment of denervation hypersensitivity permits these components of the autonomic nervous system to be individually measured by a simple, noninvasive and accurate technique. Several pharmacological agents have been employed in the assessment of autonomic denervation 
hypersensitivity; $2,3,4$ the only prerequisite to the utilisation of any drug for this purpose is the preliminary determination of the normal response. Parasympathetic denervation hypersensitivity may be effectively assessed by topical application of $2.5 \%$ methacholine chloride, ${ }^{5,6}$ and sympathetic denervation sensitivity is demonstrated by the pupillary effects of $0.5 \%$ phenylephrine hydrochloride ${ }^{7}$ normal age-adjusted tolerance intervals to both agents have recently been documented..$^{6,7}$

The aim of this study was to assess autonomic nerve function in the anterior segment of the eye in patients with primary closed-angle glaucoma and primary open-angle glaucoma, by measurement of pupillary responses to pharmacological agents recognised to be indicative of autonomic denervation hypersensitivity.

\section{Patients and Methods}

After informed consent had been obtained, ocular autonomic nerve function was assessed in 44 patients with closed-angle glaucoma (mean age $66.5 \pm 10.2$ years), 20 patients with open-angle glaucoma (mean age $66.7 \pm 7.9$ years), and 40 age- and sex-matched control subjects (mean age $65.3 \pm 12.7$ years). The diagnosis of open-angle glaucoma and closedangle glaucoma was made according to established criteria ${ }^{8,9,10}$ The open-angle glaucoma group consisted of 20 consecutive new referrals to a glaucoma clinic, prior to the commencement of treatment. A comprehensive medical history was obtained from each subject, followed by systematic general medical and ocular examination; applanation tonometry was specifically not performed until after the assessment of denervation hypersensitivity. Eyes were excluded from assessment if there was a history of:

(i) ocular operations: only unoperated eyes were included in the study. In patients with closed-angle glaucoma, assessments were performed only in unoperated fellow eyes. Eyes which had closed-angle glaucoma were specifically excluded.

(ii) ocular trauma

(iii) current ophthalmic drug treatment

(iv) corneal disease

(v) dry eyes
Subjects known to have medical disorders predisposing to autonomic nerve dysfunction, or taking medication with effects on the autonomic nervous system, were similarly excluded from the study.

\section{Autonomic Denervation Hypersensitivity}

(i) Parasympathetic nervous system

Pupil diameters were recorded photographically between 9 and 11 am under standardised lighting conditions; the subject faced a surface with measured luminance of 20 apostilbs, whilst low, constant background illumination was maintained. The subjects were positioned on an ophthalmic head-rest, thereby effecting reproducible positions for photography. A scale was placed against the lower eyelid in the perpendicular plane of the iris, the subjects fixated at 6 metres, then a photograph at $\times 3$ magnification was taken of both eyes together using a Nikon F camera with Medical Nikkor $120 \mathrm{~mm}$ f4 lens and Kodak Ektachrome Professional Film (ASA 200). One drop of $2.5 \%$ methacholine chloride solution was placed in the conjunctival sac of one eye, and one drop of sterile normal saline solution concurrently placed in the conjunctival sac of the contralateral eye. In openangle glaucoma patients and control subjects the choice of the eye to be tested was selected randomly. In closed-angle glaucoma patients only fellow eyes were tested. Forty-five minutes later, a second pupil photograph was taken. Responses to $2.5 \%$ methacholine, if present, were of short duration; pupil diameters returned to normal within one and a half hours of instilling methacholine.

\section{(ii) Sympathetic nervous system}

Three days later, the assessment of sympathetic denervation hypersensitivity was performed using essentially the same technique, substituting $2.5 \%$ methacholine by $0.5 \%$ phenylephrine hydrochloride. Assessments were performed on the same eye in each subject.

\section{(iii) Pupil measurement}

The photographic slides were projected on to a white screen at five metres, and were magnified by a factor of 17 . Horizontal pupil diameters were measured directly from the screen 
to an accuracy of $\pm 0.5 \mathrm{~mm}$, and were then corrected to actual values by comparison with relative magnification of the scale. Effectively, measurement of pupil diameter was accurate to within $\pm 0.03 \mathrm{~mm}$.

Parsympathetic denervation hypersensitivity was expressed as the $2.5 \%$ methacholine ratio:

horizontal pupil diameter 45 minutes post-test

horizontal pupil diameter pre-test

Sympathetic denervation hypersensitivity was also determined by the ratio of the postto pre-test horizontal pupil diameter, and expressed as the $0.5 \%$ phenylephrine ratio.

The concurrent assessment of an age- and sex-matched control group provided effective standards for comparison. All assessments were performed on a double-masked basis.

\section{Statistical analysis}

Comparisons were made between the results of the control group and both categories of primary glaucoma separately; significance was assessed by Student's unpaired t test. Dunnett's multiple comparison procedure was applied to the levels of significance thus obtained, to prevent incorrect conclusions from multiple comparisons with a control. ${ }^{11}$ Results are expressed as mean \pm SEM.

\section{Results}

\section{(i) Parasympathetic denervation hypersensitivity}

Significantly increased pupillary constriction in response to $2.5 \%$ methacholine chloride, indicating parasympathetic denervation of the iris, was present in both closed-angle and open-angle glaucoma groups. $2.5 \%$ methacholine ratios were significantly lower in patients with closed-angle glaucoma $(0.92 \pm$ $0.01)$ than the control group $(0: 96 \pm 0.01)$ $(\mathrm{p}<0.02) .2 .5 \%$ methacholine ratios were also significantly lower in open-angle glaucoma patients $(0.84 \pm 0.04)$ than control subjects $(\mathrm{p}<0.001)$.

\section{(ii) Sympathetic denervation hypersensitivity} Significantly increased pupillary dilatation in response to $0.5 \%$ phenylephrine, indicating sympathetic denervation hypersensitivity of the iris, was also present in both major categories of primary glaucoma. $0.5 \%$ phenylephrine ratios were significantly higher in patients with closed-angle glaucoma $(1.30 \pm$ 0.03) $(\mathrm{p}<0.01)$ and open-angle glaucoma $(1.30 \pm 0.08)(\mathrm{p}<0.05)$ than the control group $(1.19 \pm 0.03)$.

\section{Discussion}

The concept of denervation hypersensitivity originated in the early years of the 20th century; in 1904, Meltzer described paradoxical pupil dilatation in response to adrenaline, ${ }^{12}$ and in 1905 Markus $^{13}$ and Anderson ${ }^{14}$ separately noted sensitivity of the denervated pupil to cholinergic drugs. Sensitisation of the denervated pupillary sphincter to acetylcholine was confirmed by Shen and Cannon in $1936 ; ;^{15}$ the principles of denervation hypersensitivity were initially proposed by Cannon in $1939^{16}$ and subsequently confirmed in a definitive treatise by Cannon and Rosenbluth in 1949. ${ }^{17}$

Assessment of denervation hypersensitivity permits accurate and objective measurement of autonomic efficacy in the anterior segment of the eye. The results of the present study have demonstrated increased pupillary responses to minute quantities of parasympathetic and sympathetic agonists in both closed-angle and open-angle glaucoma patients, compared with an age- and sexmatched control group. Although the degree of pupillary constriction in response to $2.5 \%$ methacholine was significantly higher in openangle glaucoma than in closed-angle glaucoma patients, this does not necessarily suggest a higher prevalence of neuropathy in open-angle glaucoma. Indirect assessment of nerve function by measurement of a tissue response is obviously dependent upon an intact effector-the iris musculature; in closed-angle glaucoma patients, fellow eyes may have sustained previous episodes of intermittent angle-closure, and up to $66 \%$ of fellow eyes may subsequently develop overt angle-closure. ${ }^{18,19}$ Previous episodes of intermittent angle-closure may impair iris integrity, which is a prerequisite for the indirect assessment of autonomic denervation hypersensitivity in the anterior segment. The lesser degree of pupillary constriction demonstrated 
in closed-angle glaucoma patients compared with their open-angle glaucoma counterparts may therefore reflect impaired iris function, rather than a lesser degree of autonomic neuropathy.

The presence of ocular denervation hypersensitivity in both major categories of primary glaucoma implies that autonomic neuropathy is present in the anterior segment of both open-angle and closed-angle glaucoma. The demonstration of significant ocular autonomic dysfunction in patients with open-angle and closed-angle glaucoma must be placed in perspective. An association has been demonstrated between systemic autonomic dysfunction and all major categories of primary glaucoma; systemic autonomic neuropathy may be presented in up to $58 \%$ of patients with closed-angle glaucoma,$^{20} 42 \%$ of ocular hypertensive subjects, ${ }^{21}$ and $37 \%$ of openangle glaucoma patients. ${ }^{22}$ Autonomic neuropathy is not characteristically a localised disorder; evidence of ocular autonomic neuropathy in patients with a high prevalence of systemic autonomic dysfunction is therefore not unexpected. Although there is a significant association between autonomic neuropathy and the primary glaucomas, prevalence levels of the disorder seem to be directly related to the clinical category of glaucoma. The prevalence of autonomic neuropathy in 124 patients with primary closed-angle glaucoma $(58 \%)$ was determined by comparison of results with standard normal values ${ }^{20}$ if the results are compared with ageadjusted normal tolerance limits for autonomic function tests ${ }^{23}$ - a more rigorous parameter-the prevalence of systemic autonomic dysfunction in these patients decreases to $50 \%$. The prevalence of autonomic neuropathy in 189 ocular hypertensive subjects was reported as $42 \%,{ }^{21}$ however when this group was subdivided by angle configuration into narrow-angle and wide-angle categories, the prevalence in narrow angle patients with ocular hypertension $(50 \%)$ was significantly higher than in wide-range subjects $(34 \%)$. It is therefore apparent that the prevalence of autonomic dysfunction seems to be associated with the type of glaucoma; the prevalence in closed-angle glaucoma patients $(50 \%)$ is identical to that of ocular hypertensives with nar- row angles $(50 \%)$, whilst the prevalence in open-angle glaucoma patients $(37 \%)$ is similar to that in ocular hypertensives with wide angles (34\%).

Parasympathetic denervation hypersensitivity of the iris has previously been described in patients with ocular hypertension, and parasympathetic neuropathy has been suggested as a significant predisposing factor in the pathogenesis of raised intraocular pressure. ${ }^{6}$ The effects of autonomic neuropathy on the pathogenesis of glaucoma are probably dependent upon the pre-existing anatomical configuration of the anterior chamber. In closed-angle glaucoma patients, the anterior chamber is characteristically shallow with narrow irido-corneal angles, and is therefore predisposed to angle-closure. The importance of pupil block in angle-closure is well established; ${ }^{24,25,26}$ pupil block force is a posteriorly-directed vector, inhibiting the passage of aqueous from the posterior to the anterior chamber. Pupil block is maximal at mid-dilatation, ${ }^{27}$ the recognised pupil configuration during an acute episode of angleclosure. As previously explained, pupil diameter is directly controlled by the autonomic nervous system; parasympathetic and sympathetic neuropathy in the anterior segment would produce a mid-dilated pupil, the position of maximal pupil block. In eyes with narrow angles, increase in pupil block force may precipitate angle-closure.

In patients with open-angle glaucoma and ocular hypertension, autonomic neuropathy may influence the development of raised intraocular pressure by a different mechanism. It has been proposed that autonomic dysfunction may increase intraocular pressure in subjects with ocular hypertension by impairment of aqueous outflow from the anterior chamber. ${ }^{6}$ Parasympathetic nerve stimulation increases facility of outflow by inducing ciliary muscle contraction on the scleral spur, thereby actively opening the trabecular meshwork and utilising the potential reserve outflow. In subjects with parasympathetic dysfunction, this mechanism will be impaired, and elevation of intraocular pressure may be precipitated. Ocular hypertension may progress to overt glaucoma in a significant proportion of patients, and this 
mechanism has subsequently been forwarded as an explanation for the effects of anterior segment parasympathetic neuropathy on the pathogenesis of open-angle glaucoma. ${ }^{28}$

Evidence of autonomic denervation hypersensitivity is considered a pathognomonic feature of autonomic neuropathy. Although the present study does not establish a temporal relationship between the development of autonomic neuropathy and the diagnosis of glaucoma, there are several significant features which suggest the probable sequence of events. A high prevalence of systemic autonomic neuropathy has been demonstrated in patients with closed-angle glaucoma and open-angle glaucoma; autonomic neuropathy is not characteristically a localised disorder, and ocular autonomic dysfunction almost certainly represents a feature of the generalised disorder. As one cannot postulate a mechanism whereby glaucoma may precipitate systemic autonomic neuropathy (although the converse is a feasible hypothesis), one may reasonably assume that autonomic dysfunction either precedes glaucoma, or develops concurrently as a manifestation of a similar primary aetiological process. This does not preclude the potential secondary effects of glaucoma on ocular autonomic integrity; elevation of intraocular pressure may compromise autonomic nerve function in the anterior segment of the eye, thereby exacerbating the primary process. Thus the ocular autonomic neuropathy demonstrated by the presence of significant autonomic denervation hypersensitivity is likely to represent the effects of both primary and secondary autonomic dysfunction. The aetiology of the primary glaucomas is multifactorial; present evidence suggests that autonomic dysfunction may be a significant predisposing factor, in association with many others.

The prevalence of autonomic nerve dysfunction in each of the major categories of primary glaucoma, and also in the potentially pre-glaucomatous diagnosis of ocular hypertension, inevitably suggests a common aetiological factor in pathogenesis. Evidence of an association between autonomic neuropathy and glaucoma does not establish a causal relationship, although there are several significant factors which support this hypoth- esis. The pathogenesis of closed-angle glaucoma is dependent upon the pupil block force, which is directly controlled by the autonomic nervous system. In open-angle glaucoma, elevation of intraocular pressure occurs as a consequence of impaired aqueous outflow from the anterior chamber; as previously explained, aqueous outflow may be significantly compromised by parasympathetic neuropathy. Medical therapy in glaucoma is based upon manipulation of autonomic receptors in the anterior segment. The demonstration of significant autonomic neuropathy in the anterior segment of glaucoma patients provides a logical rationale for the efficacy of autonomic agents in the management of the disorder; therapy with autonomic agonists and antagonists may provide the autonomic control of aqueous dynamics which is inevitably impaired in a patient with autonomic neuropathy. As both parasympathetic and sympathetic pharmacological effects are specifically stimulated in the management of the primary glaucomas, one may reasonably postulate that both branches of the autonomic nervous system may be involved in the pathological process. It is significant that the same medications are effective in open-angle glaucoma and closed-angle glaucoma, although different anatomical mechanisms are involved in the genesis of both conditions, suggesting that although these conditions produce sufficiently elevated intraocular pressure to damage the retinal nerve fibre layer by different physical processes, a similar aetiological factor may be involved in the pathogenesis of both conditions.

\section{References}

${ }^{1}$ U'Pritchard DC and Snyder SH: Increase in alphareceptor number in reserpine sensitivity in rats. Eur J Pharmacol 1978, 51: 145-55.

2 Scheie HG: Site of disturbance in Adie's syndrome. Arch Ophthalmol 1940, 24: 225-37.

3 Jaffe NS: Localisation of lesions causing Horner's syndrome. Arch Ophthalmol 1950, 44: 710-28.

${ }^{4}$ Thompson HS and Mensher JH: Adrenergic mydriasis in Horner's syndrome. $A m$ J Ophthalmol 1971, 72: 472-80.

${ }^{5}$ Scheie HG and Alder FH: Site of disturbance of tonic pupils (Adie's syndrome). Arch Ophthalmol 1940, 24: 1041-4.

${ }^{6}$ Clark CV and Mapstone R: Parasympathetic dener- 
vation hypersensitivity of the iris in ocular hypertension. Invest Ophthalmol Vis Sci 1987, 28: 1732-4.

${ }^{7}$ Clark CV: Ocular autonomic nerve function in proliferative diabetic retinopathy. Eye 1988, 2: 96-101.

${ }^{8}$ Hollows FC and Graham PA: Intra-ocular pressure, glaucoma, and glaucoma suspects in a defined population. Br J Ophthalmol 1966, 50: 570-86.

${ }^{9}$ Schwartz B: Primary open-angle glaucoma In: Duane TD, Jaeger EA, eds. Clinical Ophthalmology Philadelphia: Harper and Row, 1982, 3: 52.

10 Simmons RJ and Dallow RL: Primary angle-closure glaucoma In: Duane TD, Jaeger EA, eds. Clinical Ophthalmology Philadelphia: Harper and Row, 1982, 3: 53.

${ }^{11}$ Dunnett CW: New tables for multiple comparisons with a control. Biometrics 1964, 20: 482-91.

12 Meltzer SJ and Auer CM: Studies of the 'paradoxical' pupil dilatation caused by adrenaline. Am J Physiol 1904, 11: 28-39.

${ }^{13}$ Markus C: Notes on a peculiar pupil phenomenon in cases of partial iridoplegia. Trans Ophthalmol Soc UK 1905, 26: 50-8.

${ }^{14}$ Anderson HK: The paralysis of involutary muscle. 2. On paralysis of the sphincter of the pupil with special reference to paradoxical constriction and functions of the ciliary ganglion. J Physiol 1905, 33: $156-74$.

${ }^{15}$ Shen HC and Cannon WB: Sensitisation of the denervated pupillary sphincter to acetylcholine. Chinese J Physiol 1936, 10: 359-72.

${ }^{16}$ Cannon WB: A law of denervation. Am J Med Sci 1939, 198: 737-50.

${ }^{17}$ Cannon WB and Rosenbluth A: The supersensitivity of denervated structures: a law of denervation. New York: Macmillan, 1949.

${ }^{18}$ Bain WES: The fellow eye in acute closed angle glaucoma. Br J Ophthalmol 1957, 41: 193-8.

${ }^{19}$ Mapstone R: The fellow eye. Br J Ophthalmol 1981, 65: 410-13.

${ }^{20}$ Clark CV and Mapstone R: Autonomic neuropathy in closed-angle glaucoma. Res Clin Forums 1985, 7: $45-50$.

${ }^{21}$ Clark CV and Mapstone R: Autonomic neuropathy in ocular hypertension. Lancet 1985, ii: 185-7.

${ }^{22}$ Clark CV and Mapstone R: Systemic autonomic neuropathy in open-angle glaucoma. Doc Ophthalmol 1986, 64: 179-85.

${ }^{23}$ Clark CV and Mapstone R: Age-adjusted normal tolerance intervals for cardiovascular autonomic function tests in the elderly. Age and Aging 1986, 15: 221-9.

${ }^{24}$ Barkan O: Etiology of narrow-angle glaucoma. Am J Ophthalmol 1953, 36: 901-6.

${ }^{25}$ Barkan O: Narrow-angle glaucoma. Am J Ophthalmol 1954, 37: 332-50.

${ }^{26}$ Chandler PA: Narrow-angle glaucoma. Arch Ophthalmol 1952, 47: 695-716.

${ }^{27}$ Mapstone R: The syndrome of closed-angle glaucoma. Br J Ophthalmol 1976, 60: 120-3.

28 Jordan K, Perry SR, McCormack PJ, Downes RN: Cholinergic supersensitivity of the iris in primary open angle glaucoma. Eye 1988, 2: 233-7. 